\title{
A Genetic-Algorithm-Based Fusion System Optimization for 3D Image Interpretation
}

\author{
Lionel Valet ${ }^{1}$, Beatriz S.L.P. de Lima ${ }^{2}$, and Alexandre G. Evsukoff ${ }^{2}$ \\ 1 LISTIC - Université de Savoie, BP 806, 74016 Annecy Cedex, France \\ lionel.valet@univ-savoie.fr \\ 2 COPPE/Federal University of RJ, P.O. Box 68506, \\ 21941-972 Rio de Janeiro, Brazil \\ bia@coc.ufrj.br
}

\begin{abstract}
Information fusion systems are complex systems with many parameters that must be adjusted to obtain interesting results. Generally applied in specialized domains such as military, medical and industrial areas, these systems must work in collaboration with the experts of the domains. As these end-users are not specialists in information fusion, the parameters adjustment becomes a difficult task. In addition, to find a good set of those parameters is a hard and time consuming process as the search space is very large. In order to overcome this issue a genetic algorithm is applied to automatically search the best parameter set. The results show that the proposed approach produces accurate levels of the global performance of the fusion system.
\end{abstract}

\section{Introduction}

Cooperative fusion systems devoted to image interpretation are more and more complex 11. These systems help experts in the difficult task of image interpretation which generally consists in detecting typical regions within the images. Fusion systems are composed of several steps. The first step concerns the extraction of a piece of pertinent information from the original image. Several image processing techniques could be used to characterize the different sought-after regions. Then the extracted information must be represented into a common and commensurable space in order to be aggregated in the following step. Finally, the output is expressed in an understandable space for the end-user. This step is achieved by the representation step.

Such systems generally imply in high computational cost. They also have many parameters that are not easy to use and to adjust by the end-users. The parameter setting and attributes selection are strongly necessary to obtain relevant results. Unfortunately, the end-users of this kind of fusion system are not specialists in computer sciences and they need help to interact with the system. This is reinforced by the fact that an optimized adjustment obtained for a given data is not compulsory the best one for other data.

The work presented in this paper is based on a local evaluation of the mission of the fusion system extraction step. The local evaluation turns possible to have

I. Bloch and R.M. Cesar, Jr. (Eds.): CIARP 2010, LNCS 6419, pp. 338-345, 2010.

(C) Springer-Verlag Berlin Heidelberg 2010 
a better identification of which extractor need to be adjusted. In a context of image interpretation, the extractors are based on image processing algorithms. They have technical parameters (filter coefficient, windows size, normalization, ... ) that are not accessible to the end-user. However, the parameters must be adjusted and adapted to the input image and to the sought-after regions. The high computation time makes a manual setting very difficult even for experimented users in image processing techniques. In order to solve this problem, this paper employs a local evaluation combined to the use of genetic algorithms. In the literature, GA has already shown their interests for image processing. The obtained results show that it is an interesting way to automatically optimize parameters that should have some impact on the fused result.

\section{Fusion System for 3D Image Interpretation}

\subsection{The Studied Fusion System}

The cooperative fusion system concerned in this paper, was designed for $3 \mathrm{D}$ gray level image interpretation. This application concerns the analysis of electrotechnical parts manufactured by Schneider Electric Company. The studied parts are mainly composed of glass fibers mixed with an organic matrix. The quality of the parts is directly correlated to the fiber organization. Experts (geophysics, part designers, ... ) try to understand the inside part organization to find the best fabrication process (fiber length, injection point, baking time, ... ). The method chosen by Schneider Electric to analyze the parts is based on X-ray computed tomography (CT). It is a reliable non-destructive evaluation technique. The CT results are $3 \mathrm{D}$ gray-scale images which provide data about the organization of the internal morphology. The sought-after regions are presented on figure 1 . The first one is the oriented region (noted $R_{1}$ ) which has a regular and organized texture with a single preferential orientation of the glass fibers. They are made up of long white fibers giving the impression of a flow. The Disordered regions (noted $R_{2}$ ) do not appear organized on the images, locally "chaotic", i.e. for which there is not a clearly defined principal orientation. The regions called Lack

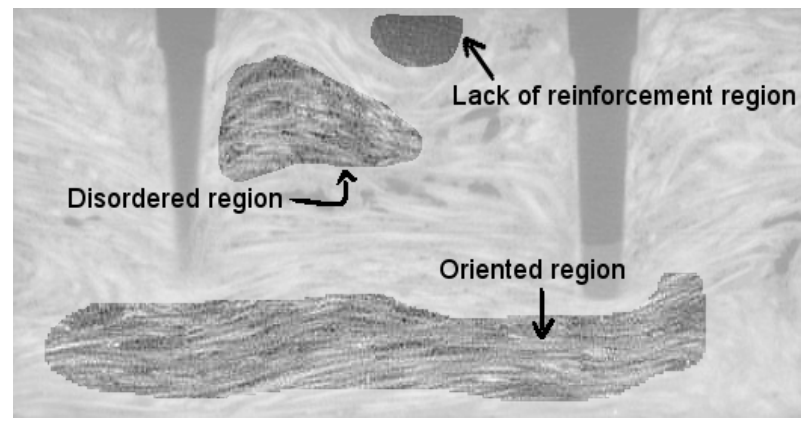

Fig. 1. The sought-after regions 
of reinforcement (noted $R_{3}$ ) only contain resin (or paste) and no glass fibers. They appear in clear and homogeneous gray level on the images. These three regions need different measurements to be detected simultaneously (orientation, texture, morphology, ... ). An information fusion system has been developed to aggregate the heterogeneous measurements.

In the concerned application, the experts introduce their knowledge by pointing references of the regions directly on the input image. To help them in the interpretation of the images (i.e. to detect the sought-after regions in all the images based on the pointing reference), the synoptic of the designed fusion system is presented on figure 2. The extraction steps involve three image processing measurements: local organisations based on principal component analysis of voxel intensity, texture measurements based on coocurrence matrix and morphological measurements based on morphology mathematics. The extracted information is then transformed into similarity map using possibility theory. Then, Choquet integral aggregates the similarity maps into a global one. The final decision on the belonging region of each voxel is obtained by a thresholding operation. The voxels that do not have enough similarity to any of the sought-after regions are labelled to a rejected class. More details on the system can be found in [2].

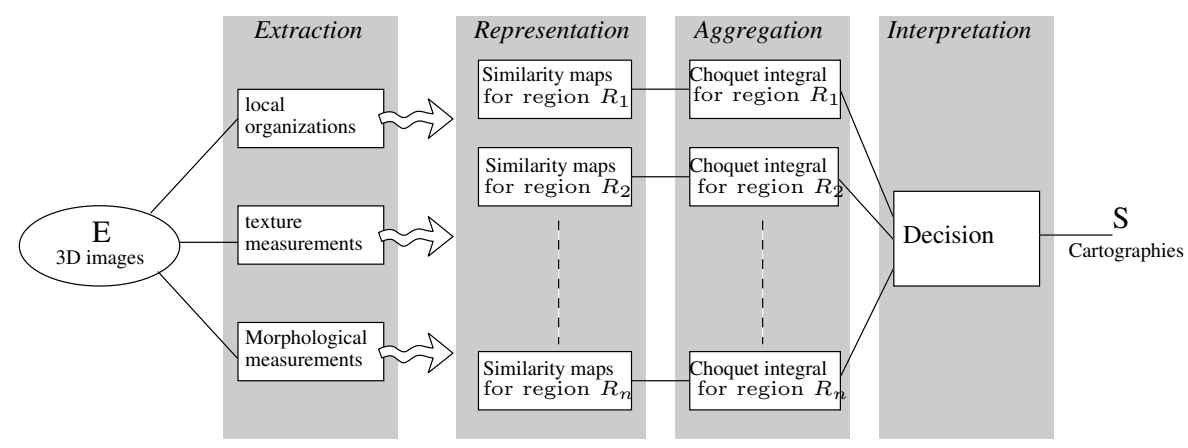

Fig. 2. Fusion system designed for 3D image analysis

In this application, the global evaluation of the system consists in verifying the correct decision of the voxels contained into the reference regions by computing a confusion matrix. Detection rate for each region (and a global one) are extracted from the matrix and serve to comment the global performance of the system. The global evaluation of the fused image does not provide enough explanation on the efficiency of each step. It is also difficult to explain the influence and the dependency of a parameter directly on the global result. Therefore there is a need for a local measure to adjust the parameters. A local evaluation of the extraction step was proposed in [4]. It is based on a mission achievement evaluation: an extracted information must bring a better separability between the sought-after regions. Separability indexes was proposed to detect weak informative attributes. 


\subsection{Importance of the Extraction Step}

In the studied fusion system devoted to image interpretation, the extraction step have a great impact on the rest of the system. Indeed, most parameters are concentrated on this step. These parameters are difficult to adjust by a end-user not specialist in image processing. A first fusion of two attributes is proposed to illustrate the complexity of the system. The first attribute noted $A_{1}$ belongs to the local organisations family. It measures the main orientation of the fiber in the part. Its computation need the following parameter: $\alpha$ the Derich filter coefficient, $W_{x}, W_{y}, W_{z}$ the window size of the principal component analysis, $W_{g}$ the window size of the gradient calculation, $N$ a normalisation coefficient. The second attribute noted $A_{2}$ belongs to the texture measurement family. The homogeneity index is computed on the coocurrence matrix evaluated on a windows $W_{x}, W_{y}, W_{z}$ and for a direction vector $\left(d_{x}, d_{y}, d_{z}\right) . A_{1}$ and $A_{2}$ were initially computed with the default parameters presented in table 1 with their separability indexes for each attribute. The separability indexes are all weak which mean that those attributes were not able to discriminate the regions. Only $A_{2}$ reached a better separability for region $R_{3}$. The detection rates are also weak even for the third region which is the best one detected. Therefore, the global detection rate is clearly insufficient.

Figure 3 presents the output cartography. White voxels correspond to region $R_{3}$, clear gray level voxels correspond to region $R_{2}$, dark grey level voxels correspond to region $R_{1}$. Black voxel are voxel belong to the rejected class because they have a weak similarity to the sought-after regions. The contour of the reference regions are also plot on the image and the hole in the part are hatched. This result clearly shows the weakness of the detection. Region are too fragmented and don't correspond to the result expected by the end-users.

To improve the detection, parameters of attribute $A_{1}$ are adjusted manually: the windows size used in the principal component analysis is increased and the $\alpha$ coefficient is decreased (growth of the smoothing effect). The new attribute is noted $A_{1}^{\prime}$ and the new separability indexes and detection rate are also presented on the table 1. Separability indexes have a small increase but not significantly. At the end, we even notice a slight improvement of the global rate, however, not considerable. The obtained cartography have also the same weakness. Manually it is extremely difficult and time-consuming to find interesting parameter according to the sought-after regions. The search space of the fusion system parameters is very large which describes a complex combinatorial optimization problem.

Table 1. Initial fusion

Attribute parameters

\begin{tabular}{|c|cccccc|}
\hline & $\alpha$ & $W_{x}$ & $W_{y}$ & $W_{z}$ & $W_{g}$ & $N$ \\
\hline$A_{1}$ & 0.5 & 9 & 9 & 9 & 15 & 10.0 \\
$A_{1}^{\prime}$ & 0.4 & 11 & 11 & 11 & 15 & 10.0 \\
\hline \hline & $W_{x}$ & $W_{y}$ & $W_{z}$ & $d_{x}$ & $d_{y}$ & $d_{z}$ \\
\hline$A_{2}$ & 8 & 8 & 8 & 2 & 2 & 2 \\
\hline
\end{tabular}

Separability indexes and detection rates

\begin{tabular}{|r|c|c|c|c|}
\cline { 2 - 5 } \multicolumn{1}{c|}{} & $R_{1}$ & $R_{2}$ & $R_{3}$ & $T_{\text {Global }}$ \\
\hline$S_{A_{1}}$ & 0,32 & 0,35 & 0,66 & \\
\hline$S_{A_{1}^{\prime}}$ & 0,35 & 0,37 & 0,67 & \\
\hline$S_{A_{2}}$ & 0,22 & 0,30 & $\mathbf{0 , 7 4}$ & \\
\hline Fusion $\left(A_{1}, A_{2}\right)$ & 0,45 & 0,69 & $\mathbf{0 , 7 4}$ & 0,52 \\
\hline Fusion $\left(A_{1}^{\prime}, A_{2}\right)$ & 0,51 & 0,53 & 0,77 & 0,54 \\
\hline
\end{tabular}




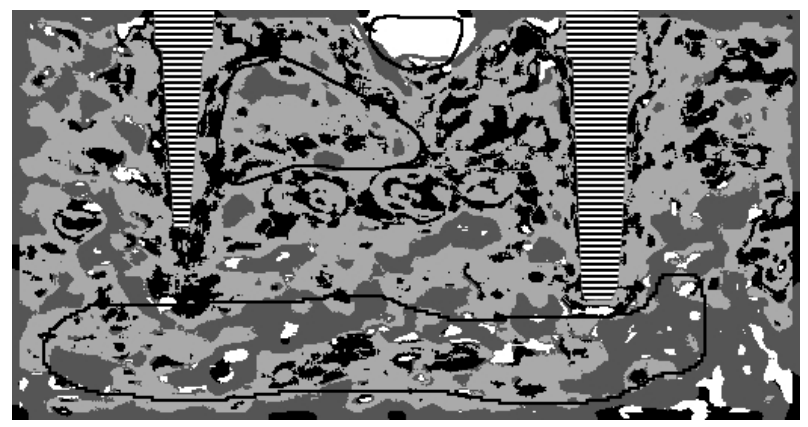

Fig. 3. Classification obtained with attributes $A_{1}$ and $A_{2}$

\section{Genetic Algorithms}

Evolutionary Algorithms (EA) have been considered as powerful search and optimization methods that prevail over the drawbacks of classical mathematical optimization methods. They are based in probabilistic searching, and do not need gradient information; therefore, they are more robust in locating global optima in multi-modal search space [5]. EAs have been widely applied in the last few years in computationally expensive applications and proved to be a strong optimization method in many types of combinatorial problems. Those successful applications of EAs involve scheduling [6], knowledge discovery [7], information fusion [8], etc.

\subsection{Principle of Genetic Algorithm}

Genetic Algorithms (GA) belong to the class of EA, it is a population-based model that uses various operators to evolve, such as selection, crossover and mutation. These operations correspond, respectively, to the principles of survival of the fittest; recombination of genetic material, and mutation observed in nature, following the mechanisms of natural selection [9].

In GAs, each optimization variable or parameter $\left(x_{n}\right)$ is encoded by a gene using an appropriate representation, such as a real number or a string of bits. The corresponding genes for all parameters $x_{1}, x_{2}, \ldots, x_{n}$ form a chromosome, capable of describing an individual design solution. A set of chromosomes representing several individual solutions comprises a population, where the fittest individuals are selected to mate and reproduce. Mating is performed using crossover to combine genes from different parents to produce children (offspring). The children inherit features from both parents, and may be submitted to mutation, which confers some truly innovative features as well. The offspring are made to compete with each other, and possibly with their parents. Individuals are evaluated via the objective function that defines the problem. As a result of the evaluation, they are assigned a certain cost that dissociate them. This value, named fitness value, represents the quality of the solution. By the end of a generation, only the fittest individuals are selected to continue in the population, and the other ones are rejected. 
Improvement in the population arises as a consequence of the repeated selection of the best parents, which are in turn more likely to produce good offspring, and the consequent elimination of low-performers. In the present work, the classical Genetic Algorithm is used, with binary codification, single point crossover, individual elitism and roulette-wheel selection with Genesis package1.

\subsection{Application to Image Processing Parameter Adjustments}

In this work, GA was applied to optimize extraction step corresponding to attribute $A_{1}$ which has the weak separability for regions $R_{1}$. Genes must be declared and configured to well represent the image processing parameters. Six genes were used, one for each of the six parameters. Chromosomes $x_{i}$ of the population are binary strings composed by the 6 genes. The gene declaration is presented in table 2. Chromosomes $x_{i}$ are composed of 26 binary elements. For the window sizes, values can only be odd. The min and max value and the number of binary elements was selected in consequence. The attribute is thus obtained by a function of the chromosomes $x_{i}: A_{1}=g\left(x_{i}\right)$ The objective function $f$ is the separability index presented in section 2 for a given region. $f:\left(A_{1}, R_{i}\right) \rightarrow S_{R_{i}}$. This function is directly dependent to the $x_{i}$ chromosomes. In this work, the main objective is to find the best set of parameters in the extraction step of image interpretation that maximizes the separability of the regions.

Table 2. Genes definition for attribute $A_{1}$

\begin{tabular}{|l|c|c|c|c|c|c|}
\hline parameters & $\alpha$ & $W_{x}$ & $W_{y}$ & $W_{z}$ & $W_{g}$ & $N$ \\
\hline genes & 1 & 2 & 3 & 4 & 5 & 6 \\
\hline binary string size & 5 & 4 & 4 & 4 & 4 & 5 \\
\hline parameter values & {$[0,1.0]$} & $\{3 . .33\}$ & $\{3 . .33\}$ & $\{3 . .33\}$ & $\{3 . .33\}$ & {$[0,200.0]$} \\
\hline number of possible values & 32 & 16 & 16 & 16 & 16 & 32 \\
\hline
\end{tabular}

\subsection{Illustration on 3D Tomography Image Interpretation}

The optimization is applied on attribute $A_{1}$ to improve the separability of this attribute to region $R_{1}$ which has the lowest detection rate. The population size of the GA is 20 individuals, the crossover Rate is set to 0.8 and the mutation Rate equal 0.01. Stopping criteria is maximum number of evaluations. The initial population is set randomly.

In this population, several individuals makes a complete separability of the region $R_{1}$ possible. Moreover, the final population also brought interesting information on the behavior of the parameters according to the sought-after regions. It shows that the Derich filter must be weak (a strong smoothing is necessary) and the gradient window size must also be weak (a windows size of 3 is enough).

\footnotetext{
${ }^{1}$ Genesis package can be found: http://www.cs.cmu.edu/afs/cs/project/airepository/ai/areas/genetic/ga/ systems/genesis/0.html
} 
Table 3. Classification obtained after optimization

\begin{tabular}{|lllllll|}
\hline$\alpha$ & $W_{x}$ & $W_{y}$ & $W_{z}$ & $W_{g}$ & \multicolumn{4}{c|}{$S_{R_{1}}$} \\
\hline $\mathbf{0 . 0 3}$ & 19 & $\mathbf{3 1}$ & $\mathbf{1 1}$ & $\mathbf{3}$ & $\mathbf{1 0 . 0 0}$ & $\mathbf{1 . 0 0 0 0}$ \\
\hline & & $R_{1}$ & $R_{2}$ & $R_{3}$ & $T_{\text {Global }}$ \\
\hline & $S_{A_{1}^{\prime \prime}}$ & $\mathbf{1 , 0}$ & $\mathbf{0 , 9 1}$ & 0,77 & \\
\hline & $\mathbf{F u s i o n} A_{1}^{\prime \prime}, A_{2}$ & $\mathbf{0 , 9 3}$ & 0,94 & $\mathbf{0 , 8 1}$ & 0,92 \\
\hline
\end{tabular}

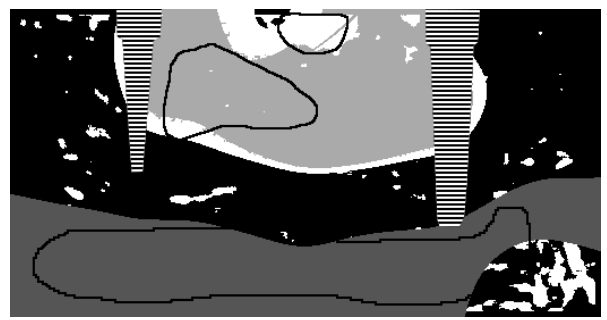

Concerning the window sizes principal component analyse, a large one $([31,33])$ is required for $W_{y}$, and finally more classical sizes have been encountered for $W_{z}$ and $W_{x}$. Values for these two parameters are also established on a larger interval. It also means that these two parameters have less influence on the result.

The parameters for the optimized attribute (noted $A_{1}^{\prime \prime}$ in the following), its separabilities and the obtained cartography are given in figure 3 . The fusion of the optimized attribute $A_{1}^{\prime \prime}$ with $A_{2}$ gives now good detection rates. The classification better presents a good correspondence to the sought-after regions and the detected regions are less fragmented. Many voxels remain classified into the rejected class (black voxel). It is an effect to the strong learning realized on the reference regions. It could have a perverse effect on the generalization of the classification (i.e. classification of the voxel for which we have no reference).

\section{Conclusions}

Cooperative fusion system for image interpretation are now complex systems. The complexity concerns both the system conception (choice of the inputs, choice of the aggregation function, ... ), the performance evaluation and the parameter adjustment. Based on an existing fusion system, this paper has proposed a way to locally adjust some parameters which could have a strong impact on the fused results. To help the end-users in the difficult task of the parameter adjustment, an optimization algorithm was proposed. Based on Genetic Algorithms, a set of best parameters can be found in a large search space. This algorithm appears interesting to find a stable local optimum that corresponds to a maximization of the objective function. Even though a set of possible interesting solution corresponding to different optima certainly exists, the main objective remains to find, at least, one situation that can improve the attribute. GA are specially interesting in this situation.

The proposed approach was applied on an attribute devoted to organisation measurement in the 3D images. The obtained parameters make it possible to have the maximum separability for a given sought-after region. Thanks to the new attributes, the global detection on the output of the fusion system can immediately considerably increase. The final parameter population brought information on the parameters (difference between the 3D dimension, variability of several one, unique value for other ones, ...). They also would have been 
difficult to find manually. However, the success of such an optimization depends on two important factors: the objective function and the reference regions. The objective function must be pertinent for the application because it corresponds to the criteria to optimize. Work is under progress to improve the actual objective function to try and attempt to optimize attribute parameters according to the 3 sought-after regions (or only one without saying which one). The reference regions are also very important. They must be representative to the sought-after regions to keep interesting results in generalization.

Acknowledgment. The authors acknowledge financial support from the CAPES/COFECUB which made the collaboration possible. We also would like to thank Schneider Electric. The fusion system concerned in this paper has been fully developed in collaboration with the material research Laboratory of Grenoble.

\section{References}

1. Kokar, M.M., Tomasik, J.A., Weyman, J.: Formalizing classes of information fusion systems. Information Fusion 5(3), 189-202 (2004)

2. Jullien, S., Valet, L., Mauris, G., Bolon, P., Teyssier, S.: An attribute fusion system based on the choquet integral to evaluate the quality of composite parts. IEEE Trans. On Instrumentation and Measurement 57(4), 755-762 (2008)

3. Zhang, Y.: A survey on evaluation methods for image segmentation. Pattern Recognition 29(8), 1335-1346 (1996)

4. Lamallem, A., Valet, L., Coquin, D.: Local versus global evaluation of a cooperative fusion system for 3d image interpretation. In: International Symposium on Optomechatronic Technologies cdrom (2009)

5. Hammouche, K., Diaf, M., Siarry, P.: A comparative study of various meta-heuristic techniques applied to the multilevel thresholding problem. Engineering Applications of Artificial Intelligence (to appear)

6. Talbi, E., Geneste, L., Grabot, B., Previtalia, R., Hostachy, P.: Application of optimization techniques to parameter set-up in scheduling. Computers in Industry 55, 105-124 (2004)

7. Gabrys, B., Ruta, D.: Genetic algorithms in classifier fusion. Applied Soft Computing 6, 337-347 (2006)

8. Maslov, I., Gertner, I.: Multi-sensor fusion: an evolutionary algorithm approach. Information Fusion 7, 304-330 (2006)

9. Goldberg, D.: Genetic Algorithms in Search, Optimization and Machine Learning. Addison-Wesley, Reading (1989)

10. Chaibakhsh, A., Ghaari, A., Moosavian, S.A.A.: A simulated model for a oncethrough boiler by parameter adjustment based on genetic algorithms. Simulation Modelling Practice and Theory 15, 1029-1051 (2007)

11. Montero, G., Rodrguez, E., Montenegro, R., Escobar, J., Gonzalez-Yuste, J.: Genetic algorithms for an improved parameter estimation with local renement of tetrahedral meshes in a wind model. Advances in Engineering Software 36, 3-10 (2005)

12. Nougues, J.M., Grau, M.D., Puigjaner, L.: Parameter estimation with genetic algorithm in control of fed-batch reactors. Chemical Engineering and Processing 41, 303-309 (2002) 